Rep. 4, Bur. Gravimétrique Int., Toulouse, France, 1982

Balmino, G., C. Brossier, A. Cazenave, and F Nouel, Geoid of the Kerguelen Islands area determined from GEOS 3 Altimeter data, J. Geophys. Res., 84, 3827, 1979.

Cazenave, A., B. Lago, and K. Dominh, Thermal parameters of the oceanic lithosphere estimated from geoid height data, $J$ Geophys. Res., 88, 1105, 1983.

Haxby, W. F., G. D. Karner, J. F. Labrecque, and J. K. Weissel, Digital images of combined oceanic and continental data sets and their use in tectonic studies, Eos Trans. $A G U, 64,995,1983$

Lazarewicz, A. R., and D. C. Schwank, Detection of uncharted seamounts using satellite altimeter data, Geophys. Res. Lett., 9, 385, 1982

Lerch, F. J., B. H. Putney, C. A. Wagner, and S. M. Klosko, Goddard earth models for oceanographic applications (GEM 10B and 10C), Mar. Geodesy, 5, 145, 1981

Levitus, S., Climatological Atlas of the World Ocean, NOAA Prof. Publ. 13, Geophys. Fluid Dyn. Lab., Princeton, N.J., 1982.

Marsh, J. G., and T. V. Martin, The Seasat altimeter mean sea surface model, $J$ Geophys. Res., 87, 3269, 1982.

Marsh, J. G., A. Brenner, B. D. Beckley, and T. V. Martin, Global mean sea surface computation using GEOS 3 altimeter data, J. Geophys. Res., 87, 955, 1982

Marsh, J. G., R. E. Cheney, J. J. McCarthy, and $T$. V. Martin, Regional mean sea sur faces based upon GEOS 3 and Seasat altim eter data, Mar. Geodesy, 8, 385, 1984.

Rapp, R. H., Global anomaly and undulation recovery using GEOS 3 altimeter data, $O S U$ Rep. 285, Dep. of Geodetic Sci. and Survey ing, Ohio State Univ., Columbus, 1979.

Rapp, R. H., The earth gravity field to degree and order 180 using Seasat altimeter

data, terrestrial gravity and other data, Rep 322, Dep. of Geodetic Sci. and Surveying, Ohio State Univ., Columbus, 1982.

Rapp, R. H., The determination of geoid undulations and gravity anomalies from Seasat altimeter data, J. Geophys. Res., 88, 1552 , $1983 a$.

Rapp, R. H., The development of the January $19831^{\circ} \times 1^{\circ}$ mean free-air anomaly data tape, internal report, Dept. of Geodet ic Sci. and Surveying, Ohio State Univ., Columbus, $1983 b$.

Reigber, C., H. Müller, W. Bosch, G. Balmino, and B. Moynot, GRIM gravity mode improvement using LAGEOS (GRIM 3 L1), J. Geophys. Res., 90, 9285, 1985.

Torge, W., G. Weber, and H. G. Wenzel, High-resolution gravimetric geoid heights and gravimetric vertical deflections of Europe including marine areas, Mar. Geophys. Res., 7, 447, 1984

Georges Balmino graduated from the Ecole Normale Supérieure of St. Cloud and then received $a$ master's degree in mathematics and a Ph.D. in physics (in 1973) from Paris University. After several years with the Meudon Astronomical Observatory. he joined the Centre $\mathrm{Na}$ -

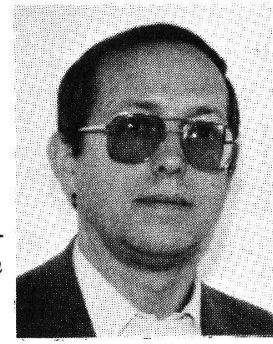
tional d'Etudes Spatiales in Toulouse (1974), where he led the French part of the team involved in the Franco-German GRIM geopotential models. Balmino has been director of the Bureau Gravimétrique International since 1980. His current research interests involve celestial mechanics, satellite geodesy, and planetology.
Bernard Moynot received his degree in mathe matics from the Ecole Nor male Supérieure of Paris in 1963. In 1956, he be gan working with the Centre National d'Etudes Spatiales, performing research in satellite geodesy and numerical analysis. $\mathrm{He}$ is now with the Bureau Gravimétrique International in Toulouse.

Michel Sarrailh graduated from the Institut de Physique du Globe in Strasbourg, France, and received an engineering de gree in geophysics in 1971 After working for 2 years with the SAPA Geophysical Company, he taught geophysics at the Institut Algérien du Pétrole (Algeria)

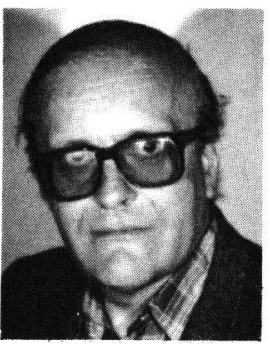
and then joined the Paris Institut de Physique du Globe in 1978. Since 1980, Sarrailh has been working with the Bureau Gravimétrique International in Toulouse.

Nicole Valès received degrees in physics and chemistry from Toulouse University in 1972. She then worked in computer sciences at Informatique Internationale. Since 1981 , she has been with the Centre National d'Etudes Spatiales. Valès is involved with earth and

planetary science computations at the Groupe de Recherches de Géodésie Spatiale, a department of the Centre National d'Etudes Spatiales that hosts the Bureau Gravimétrique International.

\title{
CEDAR:
}

\section{An Aeronomy Initiative}

PAGES 19-21

\section{G. J. Romick, ${ }^{1}$ T. L. Killeen, ${ }^{2}$ D. G. Torr, ${ }^{3}$ B. A. Tinsley, ${ }^{4,5}$ and R. A. Behnke}

\section{Introduction}

A 3-year process of committee deliberations and community-wide annual workshops by scientists involved in aeronomical studies has led recently to the presentation of a final report to the National Science Foundation,

${ }^{1}$ Geophysical Institute, University of Alaska, Fairbanks

${ }^{2}$ Space Physics Research Laboratory, University of Michigan, Ann Arbor

${ }^{3}$ Department of Physics, University of Alabama at Huntsville

${ }^{4}$ Center for Space Sciences, University of Texas at Dallas, Richardson

${ }^{5}$ Division of Atmospheric Sciences, National Science Foundation, Washington, D.C. outlining a comprehensive 7-year plan for the ground-based study of the earth's upper atmosphere. The two-volume report, entitled "CEDAR: Coupling, Energetics, and Dynam ics of the Atmospheric Regions," was assembled under the aegis of the Aeronomy Program of NSF and involved the efforts of a team of 66 active researchers from 32 U.S. and Canadian institutes. The volumes, taken together, contain a review of the present state of the art in current and projected experimental and theoretical capabilities for the field, ranging from the middle atmosphere (mesosphere) through the thermosphere and ionosphere and into the exosphere and protonosphere of the earth. The first volume contains the scientific rationale for a program of careful, measured instrumental develop- ment and deployment activities, coupled with related theoretical modeling work using a fully coordinated approach to a series of major outstanding scientific questions. The second volume, in two parts, contains the collated reports from the seven subcommittees charged with the specific areas of theoretical modeling, spectroscopy, interferometry, imaging, LIDAR, ionospheric/thermospheric radar, and lower-thermospheric/mesospheric radar.

The CEDAR plan is in response to the call from the National Academy of Sciences for a National Solar-Terrestrial Research Program, or NSTP (see the report "National Solar-Terrestrial Research Program" by the Committee on Solar Terrestrial Research, National Academy of Sciences, Washington, D.C., 1984). NSTP, as defined by the academy, incorporates three elements: a spaceborne component, a theory and data analysis program, and a strong program of ground-based experimental studies. CEDAR represents an important contribution to the latter two NSTP elements, as it provides a link and a complement to the upcoming NASA Upper Atmosphere Research Satellite (UARS) and the International Solar Terrestrial Program (ISTP), whose national component has been recently relabelled the Global Geospace System (GGS) The initial impetus for the CEDAR effort came from a general realization that the in- 


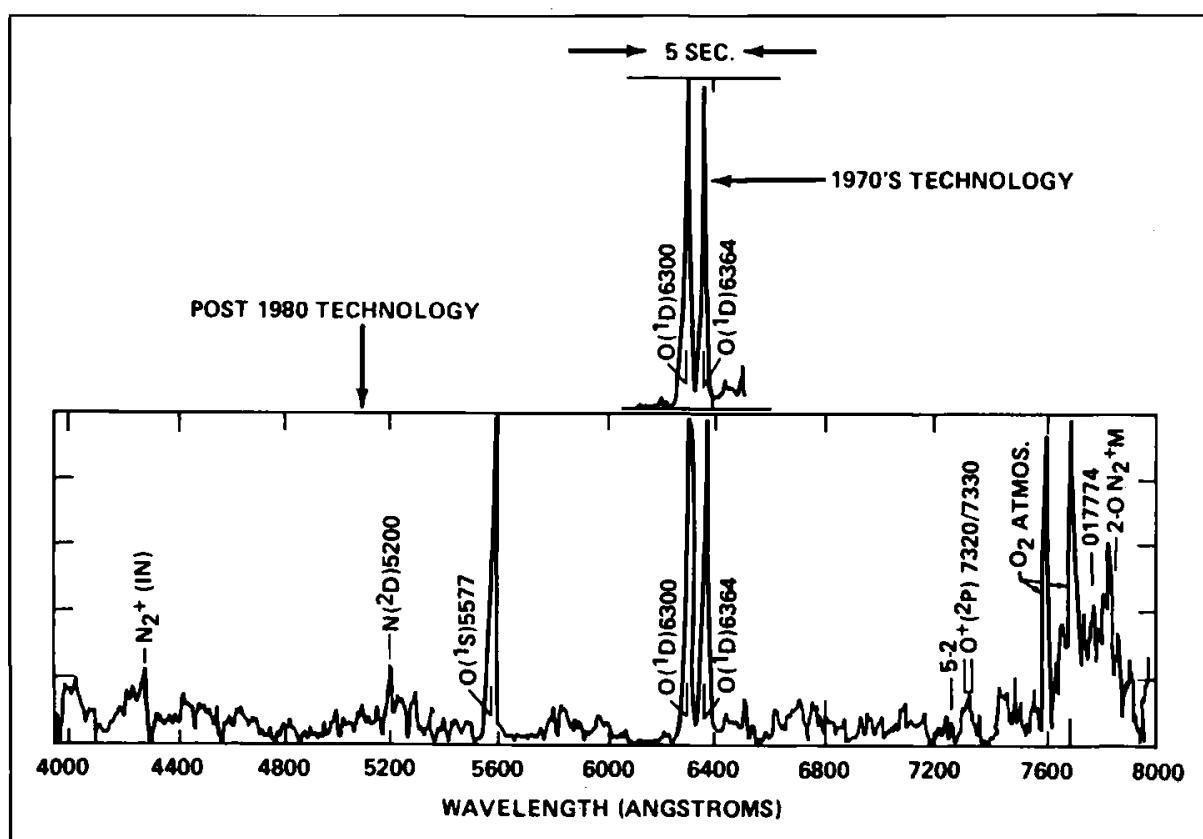

Fig. 1. Illustration of the great improvement in data-taking ability of high-resolution spectrometers afforded by modern technology. Rapid coverage of an extensive spectral region is required for many problems in quantitative atmospheric spectroscopy. Similar, dramatic improvements in the capabilities of the various optical and radar instruments used in aeronomical studies are possible by using existing technology and will provide significant observational benefits over a broad range of scientific topics in atmospheric physics and chemistry.

strumental techniques deployed in aeronomical field campaigns used technology that was 2 decades old. Experimentalists were concerned that existing modern designs and techniques were not being used to benefit ground-based aeronomy. The concern was founded on the fact that aeronomy is, in general, a "signal-limited" science in that the sensitivity of the various instruments (radar, photometers, spectrometers, imagers, interferometers, LIDAR, etc.) limits the number of feasible experiments and introduces tantalizing uncertainties in interpretations because of poor statistics. As a simple but telling example of the utility of more modern technology for aeronomy, Figure 1 illustrates the vast improvement in speed of spectrometer systems based on modern (charge-coupled device, or CCD) technology over those using more traditional single-channel phototubes. The speed and spectral range covered by such instruments can be critical for successfully attacking many problems in quantitative atmospheric spectroscopy. Another example from the radar field would be the high-speed digital signal processing currently available for the acquisition of real-time data.

From these initial experimental concerns, however, the scope of the CEDAR study was broadened as a full scientific rationale was sought to justify proposed experimental development and the use of powerful modern but relatively expensive technology. The ensuing thorough scientific review of aeronomy from the middle atmosphere through the thermosphere and ionosphere to the exosphere provided the justification for improvements in instrumentation but also pointed, perhaps more fundamentally, to a clear requirement for community-wide and even international coordination in the planning and execution of many future aeronomical studies. This last requirement represents a significant change in approach for a scientific community nurtured on the idea of single investigators contributing first-rate science from individual observatories. While this undoubtedly remains true in certain cases, many of the major outstanding scientific issues call clearly for a coordinated multistation, multiinstrument approach. The reason for the change in emphasis is related to the recent development of our understanding of the near-space environment. Previous national research efforts have focused on individual regions of the solar terrestrial system. For example, the NSF incoherent scatter radar chain has concentrated on the study of the ionosphere and thermosphere, the Solar-Mesosphere Explorer (SME) satellite on the mesosphere, the Atmosphere Explorer series on the photochemistry of the thermosphere and ionosphere, the International Sun-Earth Explorer (ISEE) series on the magnetosphere and interplanetary system, the Solar Maximum Mission (SMM) on the sun, and the Dynamics Explorer twin spacecraft on the dynamics and electrodynamics of the thermosphere. Spurred by these programs, relatively mature models of individual components of the solar-terrestrial environment exist. These include global (or global-scale) models of the troposphere, straiosphere, mesosphere, thermosphere, ionosphere, exosphere, plamasphere, magnetosphere, interplanetary medium, and the sun.

Today, the major questions posed relate to the interactions between regions: Interactions that are currently only crudely understood. Thus the principal scientific challenge before aeronomy is to understand the coupled atmospheric regions on a global scale and as a whole, including, for example, the effects of energy, momentum, and compositional interchange between regions. To meet this challenge will require not only new and improved instruments but will also necessitate globalscale coverage of many parameters, as well as measurements of the critical parameters related to the important couplings between atmospheric regions. Such measurements will require coordination and collaboration among experimentalists and between experimentalists and theoreticians. They will also require a pooling and common use of data collected from networks of observatories and facilities.

The process of reviewing both the theoretical and experimental status of aeronomy by the CEDAR subcommittees and the development of a 7-year program plan representing the consensus view of the U.S. Aeronomy Community lasted 3 years. During this period, well-attended annual summer workshops were held (at Utah State University, Logan, in 1983; at the University of Michigan, Ann Arbor, in 1984; at the University of Seattle, Seattle, Wash., in 1985; and at the High Altitude Observatory of the National Center for Atmospheric Research (NCAR), Boulder, Colo., in 1986) to ensure the full participation of the broad community. Focused steering committee and subcommittee meetings were also conducted at more frequent intervals.

This relatively lengthy review process has already created a new atmosphere of cooperation and collaboration within the aeronomy community, cooperation that is paying off in terms of significant collaborative projects. Furthermore, the many workshops and the increasingly intensive interaction between atmospheric scientists with closely related and complementary interests has led to a spirit of collegiality that bodes well for the future health and vitality of the field.

In the following sections, we provide a brief summary of the scientific rationale and the program plan for CEDAR as laid out in the report to NSF. Interested readers can obtain copies of both volumes of the report from the authors (contact G. J. Romick, Geophysical Institute, University of Alaska, Fairbanks, AK 99701).

\section{Scientific Rationale for CEDAR}

Aeronomy is the study of the earth's upper atmosphere and of the physical, chemical, and plasma processes that modulate and partition the flow of solar energy through our near-space environment. The scientifc objectives for CEDAR include the study of a broad range of problems related to the physics that couples atmospheric regions. The cover of this issue is a schematic illustration of some of the interesting phenomena that occur in the altitude range covered under the initiative, as well as some of the ground-based techniques that can be used profitably to study these and other phenomena.

The earth's upper atmosphere is constantly undergoing periodic and aperiodic changes. These changes are induced by solar energy entering the atmosphere, both directly as electromagnetic radiation and indirectly through magnetospheric convection and particle precipitation. The latter energy is impulsively released in ways that depend on the de- 


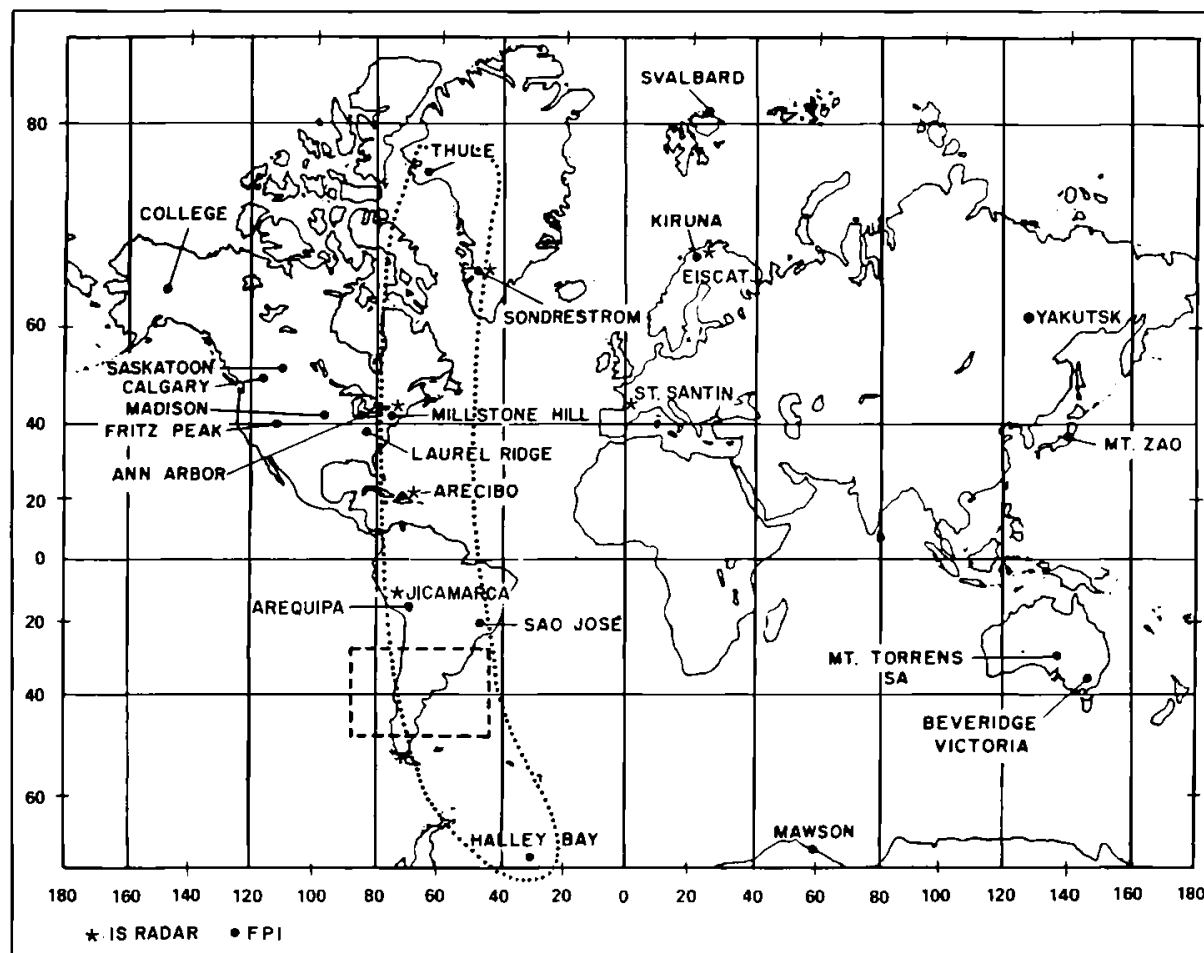

Fig. 2. Worldwide distribution of incoherent scatter (IS) radar facilities and Fabry-Perot interferometer (FPI) observatories, indicating present capabilities for coordinated measurements with, for example, the "pole-to-pole" longitudinal chain enclosed by the dotted line.

tails of how the interplanetary magnetic field merges with the geomagnetic field. This process generates complex and variable electric potentials and currents that, in turn, result in large insertions of electrical and corpuscular energy to the upper atmosphere. The consequences of this variable energy input are felt across the globe and are manifested by changed in ionization, excitation, heating, dynamical behavior, composition, and thermal structure.

In addition, thermal and orographic effects initiated at low altitudes influence the upper atmosphere. These effects force the general circulation and create wave motions of all kinds (tidal, planetary, and internal gravity).

Many of the associated aeronomic processes leave some atmospheric constituents in excited states, and their excess energy is partially shed through visible and near-infrared emissions at specific wavelengths. Groundbased measurements of these emissions provide direct information on the composition of the atmosphere, on its dynamical and thermodynamical state, and on various physical and chemical processes that occur. These emissions originate from the entire altitude range of interest, encompassing the troposphere, stratosphere, mesosphere, thermosphere, ionosphere, and magnetosphere. In addition to the use of optical emissions as a diagnostic of upper atmosphere processes, active sounding techniques employing powerful radars and LIDARs are able to measure many of the important parameters directly as a function of altitude.

As mentioned above, relatively mature theoretical models of individual components of the solar terrestrial environment exist. These models can be effectively constrained and thereby improved by using ground-based observations. For atmospheric models the major questions now posed relate to the interactions or coupling between the various atmospheric regions. The CEDAR program is commited to utilizing the existing data from satellites, rockets, radars, and optical instruments, together with data from the recommended new program of ground-based measurements, to study the coupled near-earth environment as a whole.

The missing elements of the current observational base are simultaneous measurements, on a global scale, of key parameters relevant to coupling throughout the solar terrestrial system. The program plan for CEDAR recommends the establishment of a number of strategically located observatories with a synergistic complement of instrumentation, including combinations of the following instruments:

- more sensitive spectrometers, interferometers, and imagers;

- upgraded existing large radars; and

- new and upgraded LIDAR and small radar systems.

The plan allows for a much-improved coverage of the essential observables required for the study of coupling processes. A fundamental aspect of the program will be the coordination of activities, with carefully considered instrument development, deployment, and data handling strategies.

Three broad categories embrace the scientific goals of the CEDAR program:

- Dynamics and energetics of the upper atmosphere from the mesopause upward to the exobase, with particular emphasis on the hard to observe region between 80 and $150 \mathrm{~km}$.

- Ionospheric-thermospheric-exosphericmagnetospheric coupling.

- Mesospheric-thermospheric coupling. Within these categories are many scientific topics that require detailed study. The first volume of the CEDAR report itemizes the specific science topics for study ard recommends a phased approach (discussed below) as a method of systematic investigation that would take advantage of the projected instrumental and modeling developments over the next few years. Readers are referred to the two-volume CEDAR report for further details concerning the individual topic-related scientific objectives and program implementation strategy.

\section{Phasing Strategy and Management Plan}

The approach recommends a coordinated program of research and sequential steps leading to the development and deployment of new instrumentation, involving both improved versions of present types and 1990 state-of-the-art facilities. The plan involves three phases of implementation designed to match growth in hardware and modeling development with budgetary resources. A finding of interest that emerged from the studies was the rapid increase in the number of tractable science problems that could be addressed with the evolving complement of new and improved instruments.

In Phase 1, which has already begun, utilization of the existing inventory of instruments will be optimized by introducing coordinated observational programs designed to provide data bases that would otherwise not exist. This significantly increases the number of scientific topics that can be addressed. For example, one of the first coordinated programs defined in the CEDAR report will provide the data needed to define the mean characteristic behavior of global thermospheric winds and temperatures, as well as to quantify departures from the mean. Initial campaign activities to accomplish this objective were carried out during winter 19851986 from a number of optical and radar facilities (indicated in Figure 2). A second program addressed the global-scale morphology of mesopause temperatures from a similar global network of optical facilities. A third coordinated program is providing a data base on both auroral latitude and low-latitude energy and momentum inputs, together with information on waves and dynamical perturbations in the upper atmosphere. In the latter program, as throughout the recommended CEDAR activities, the most appropriate combination of optical and radar ground-based facilities will be used.

In Phase 2, existing instruments will be upgraded, and additional observing stations will be established to fill out the global grid, expanding the capability towards fuller studies of global-scale coupling. This activity will approximately triple the number of scientific topics that can be addressed, adding, for example, studies of the effects of ring current energetic particle precipitation, studies of exospheric hydrogen, and studies of thermospheric composition, dynamics, and energetics for various geophysical conditions. The same instrument complement will provide data on dynamics, composition, and temperatures of the mesosphere, establishing the initial framework for studying the coupled thermosphere/mesosphere system. At the same time, improved measurements of auroral processes will be acquired, including the spec- 
troscopy of emissions relevant to many atomic and molecular processes and the observations of electron densities and temperatures with sufficiently high altitude and time resolution.

This increase in available high-quality information will be accompanied by the development of advanced modeling capabilities and the holding of coordinated workshops designed to address specific topics. Recent models for successful topic-related workshops include the coordinated incoherent scatter radar Global Incoherent Scatter Monitoring of Substorms (GISMOS) study and the Global Thermospheric Mapping Study (GTMS)

Phase 3 realizes the full potential of the CEDAR program, in which the maximum number of scientific topics will be addressed. With the establishment of the advanced technology "Class l" facilities, deployed at six to eight strategically-located sites, the science potential rapidly increases. In addition to the topics already mentioned, some examples of the new science to be addressed include studies of $E$ region transport, including feedback from the thermosphere to the magnetosphere; observations of the tides in the mesosphere and their propagation to the thermosphere; and studies of the mesospheric and lower thermospheric gravity wave momentum and turbulence budgets. Simultaneous LIDAR and rocket measurements will provide data on the variability of the mesospheric eddy diffusion coefficient, together with detailed information on the concentrations of ions and neutrals and on temperatures, as well as on dust and noctilucent clouds. Coordinated observations of the above type will provide a unique opportunity for studies of coupling between the regions of the upper atmosphere. The plan for this final phase of CEDAR includes full-scale integration of several instruments per observatory, all with coordinated data acquisition, processing, and rapid communal access to data, as well as to the predictions of relevant theoretical models.

A critical step in developing the group of Class 1 facilities involves their operation and management. The NSF Upper Atmosphere Facilities Program already provides an established vehicle for this activity. The CEDAR
Science Steering Committee, impanelled by the NSF Aeronomy Program, will provide scientific guidance through the various CEDAR phases. With suitable additions to technical and scientific staff, NCAR will assume responsibility for archiving and providing access to the large data bases.

\section{Summary}

The CEDAR program was developed over a 3-year period of workshops, science symposia, and committee deliberations. It attempts to provide a realistic framework for the development of upper atmospheric research in the United States through an evolutionary strategy of instrument development and deployment activities designed to meet the challenge of a set of important scientific topics related to the study of the global-scale coupled near-earth environment. The potential contribution of CEDAR studies to the national effort in solar-terrestrial research is great. There has already been substantial interest shown in CEDAR by our international colleagues, and it is to be anticipated that the collaborative approach adopted by the U.S. aeronomy community will extend to include various other national groups and organizations. In order to minimize costs for the program, the program planners envision gradual growth over several years, optimizing the scientific usage of existing capabilities and stressing the gains to be made by adopting a coordinated approach to the study of the earth's upper atmosphere.

\section{Acknowledgments}

The CEDAR study was conducted under the auspices of the NSF Aeronomy Program. Individuals from many institutes have made important contributions to the effort. A full list of the CEDAR committee and subcommittee membership can be found in volume 1 of the report.

Gerald Romick received his Ph.D. from the Geophysical Institute of the University of Alaska in 1964. He is currently an emeritus professor of geo- physics at the University of Alaska. His principal scientific interests include detailed studies of the aurora, including coordinated satellite, rocket, and ground-based spectroscopic measurements of the altitudinal structure. Romick serves as the chairman of the NSF CEDAR Science Steering Committee.

Timothy L. Killeen received his $P$ h.D. from University College London in 1975. He is currently an associate research scientist in the Space Physics Research Laboratory of the University of Michigan. Killeen's principal scientific interest is in the dynamics of the upper atmosphere. He serves on the National Academy of Sciences (NAS) Committee for Solar-Terrestrial Research, is an associate editor of the Journal of Geophysical ResearchSpace Physics, and is currently serving as secretary of the AGU Aeronomy Section.

Brian Tinsley has begun a 2-year term as program director for aeronomy at NSF. He is a professor of physics at the University of Texas at Dallas and has been involved in observational and theoretical studies of geocoronal hydrogen and of lowlatitude aurora, as well as in the imaging of transequatorial ionospheric bubbles. He served as chairman of the International Association of Geomagnetism and Aeronomy Division II during 1973-1979.

Richard Behnke received his Ph.D. in space science and aeronomy from Rice University in 1970. From that time until 1982, he worked at the National Astronomy and Ionosphere Center in Arecibo, Puerto Rico, where he studied the motions of the earth's upper atmosphere. Since 1982, he has been program manager for upper atmosphere facilities at NSF.

Douglas Torr received his Ph.D. in ionospheric physics from Rhodes University in South Africa. He is currently a professor of physics at the University of Alabama, Huntsville. While Torr has specialized in thermospheric photochemistry, his interests range from magnetosphere-ionosphere coupling to the chemistry and dynamics of the stratosphere, mesosphere, and thermosphere. He has served as editor of Geophysical Research Letters and on committees such as the NAS Committee on Solar and Space Physics and various NASA and NSF committees, including the NSF CEDAR steering and modeling committees. 